\title{
Supporting Nested Locking in Multiprocessor Real-Time Systems*
}

\author{
Bryan C. Ward and James H. Anderson \\ Department of Computer Science, University of North Carolina at Chapel Hill
}

\begin{abstract}
This paper presents the first real-time multiprocessor locking protocol that supports fine-grained nested resource requests. This locking protocol relies on a novel technique for ordering the satisfaction of resource requests to ensure a bounded duration of priority inversions for nested requests. This technique can be applied on partitioned, clustered, and globally scheduled systems in which waiting is realized by either spinning or suspending. Furthermore, this technique can be used to construct fine-grained nested locking protocols that are efficient under spin-based, suspension-oblivious or suspension-aware analysis of priority inversions. Locking protocols built upon this technique perform no worse than coarse-grained locking mechanisms, while allowing for increased parallelism in the average case (and, depending upon the task set, better worst-case performance).
\end{abstract}

\section{Introduction}

To support real-time applications on multiprocessor platforms, real-time scheduling and synchronization algorithms are required that enable timing constraints to be met. While prior scheduling-related work has produced many viable scheduling options, serious limitations remain pertaining to synchronization. Perhaps most significantly, all current stateof-the-art real-time multiprocessor locking protocols (see [2, 11] for relevant citations) directly support only non-nested shared resource requests; nested requests, which are commonly employed in practice, are only indirectly supported through the use of coarse-grained locking techniques such as group locks. A group lock treats a set of shared resources as a single resource, and arbitrates access to the group using a single-resource locking protocol [1].

Unfortunately, group locks are inflexible because resources must be statically grouped before execution. They also result in pessimistic analysis because a job waiting on one resource in a group must block while a job holds another resource in the group. This pessimism limits the degree of concurrency, which is particularly concerning in today's increasingly parallel system architectures.

Alternatively, resource groups can be broken into smaller elements that are acquired individually. This is called finegrained locking, and it is useful in a variety of settings. For example, in a shared linked list, each element can be controlled with a single lock, instead of locking the whole list.

*Work supported by NSF grants CNS 1016954 and CNS 1115284; ARO grant W911NF-09-1-0535; AFOSR grant FA9550-09-1-0549; and AFRL grant FA8750-11-1-0033.
This allows different jobs to access separate sections of the list concurrently. Nested locking protocols can also be used in applications such as real-time database systems.

To enable fine-grained locking in multiprocessor real-time systems, we discuss a family of efficient locking protocols for partitioned, clustered, and globally scheduled job-level static priority (JLSP) task systems, i.e., systems in which a job's priority is constant (e.g., as in earliest-deadline-first (EDF) or static-priority scheduling). Tasks can either wait by spinning (busy-waiting) or by suspending. We analyze locking protocols on the basis of priority inversion blocking (piblocking), i.e., the duration of time a job is blocked while a lower-priority job is running. In schedulability analysis, the execution time of a task must be inflated by the duration of piblocking to ensure timing constraints are met. Under analysis assumptions used previously [4, 2, 5], most of the locking protocols we develop are asymptotically optimal.

Prior work. The uniprocessor priority ceiling protocol $(P C P)$ has been extended to multiple processors in a number of locking protocols, for example, the multiprocessor $P C P(P C P)$ [11], distributed PCP (DPCP) [11], multiprocessor dynamic PCP (MDPCP) [6] and the parallel PCP $(P P C P)$ [7]. Of these, only the MDPCP claims to support nested resource requests, albeit in a somewhat limited fashion-the MDPCP is limited to periodic tasks scheduled by partitioned EDF, has somewhat high worst-case blocking bounds, and enables higher concurrency than group locking (with respect to nested resource accesses) only in certain corner cases. Recently, several other protocols have been developed that are asymptotically optimal for multiprocessor JLSP systems. These protocols and their blocking bounds, which are summarized in Table 1, are discussed next.

In work on optimal suspension-oriented multiprocessor locks, Brandenburg and Anderson [4] presented two definitions of pi-blocking for suspension-oriented multiprocessor locking protocols: suspension-oblivious (s-oblivious), in which suspensions are modeled as computation, and suspension-aware (s-aware), in which suspensions are accounted for. They also established a per-request lower bound on pi-blocking of $\Omega(n)$ in the s-aware case, and $\Omega(m)$ in the s-oblivious case, where $n$ is the number of tasks and $m$ is the number of processors [4].

Block et al. [1] developed the flexible multiprocessor locking protocol (FMLP), in which waiting can be realized as either spinning or suspending. Under the FMLP, a spinning or resource-holding job runs non-preemptively. Later, Brandenburg extended the FMLP to obtain the FIFO mutex locking protocol $\left(\mathrm{FMLP}^{+}\right)$, which is more flexible because it lifts this requirement [2]. Under s-aware analysis, for a large category 


\begin{tabular}{|c|c|c|c|c|}
\hline Analysis & Scheduler & Locking Protocol & Every Job Pi-blocking & Per-Request Pi-blocking \\
\hline spin & Any & FMLP/FMLP & $m L^{\text {max }}$ & $(m-1) L^{\max }$ \\
\hline \multirow{3}{*}{ s-aware } & Partitioned & FMLP & $n L^{\text {max }}$ & $(n-1) L^{\max }$ \\
\cline { 2 - 5 } & Clustered & FMLP & $O(\phi \cdot n)^{\dagger}$ & $(n-1) L^{\max }$ \\
\cline { 2 - 5 } & Global $^{\ddagger}$ & FMLP & $O(n)$ & $(n-1) L^{\max }$ \\
\hline \multirow{3}{*}{ s-oblivious } & Partitioned & P-OMLP & $m L^{\max }$ & $(m-1) L^{\max }$ \\
\cline { 2 - 5 } & Clustered & C-OMLP & $m L^{\max }$ & $(2 m-1) L^{\max }$ \\
\cline { 2 - 5 } & Global & OMLP & 0 & 0 \\
\hline
\end{tabular}

${ }^{\dagger} \phi$ is the ratio of the maximum to minimum period as discussed in Sec. 5.4. The issue of optimality is still open for the s-aware clustered case.

$¥$ Applicable only under certain schedulers as discussed in Sec. 5.4.

Table 1: Summary of existing single-resource locking protocols and their blocking complexity. The column "Every Job Pi-Blocking" indicates how long any job in the system (whether it accesses shared resources or not) can be pi-blocked when it is not currently utilizing the locking protocol to access a shared resource. The column "Per-Request Pi-Blocking" indicates how long a job can be pi-blocked per request. $L^{\text {max }}$ denotes the maximum critical section length. All listed protocols are asymptotically optimal, except as noted.

of global schedulers and all partitioned schedulers, the FMLP and/or the $\mathrm{FMLP}^{+}$are asymptotically optimal; ${ }^{1}$ their spinbased counterparts are also optimal. Brandenburg and Anderson also developed the $O(m)$ locking protocol $(O M L P)$ family of suspension-based protocols, which are asymptotically optimal under s-oblivious analysis $[4,5]$. The OMLP can be used on partitioned, clustered, and globally scheduled systems. None of these previous multiprocessor locking protocols support fine-grained lock nesting.

Contributions. In this paper, we present the first multiprocessor real-time locking protocol that supports fine-grained nested resource requests. This locking protocol, called the real-time nested locking protocol $(R N L P)$, employs a $k$ exclusion lock, ${ }^{2}$ which can be implemented in different ways, giving rise to a family of mutual exclusion (mutex) locking protocols. We show that the RNLP can be used on global, clustered, and partitioned JLSP systems and when waiting is realized through spinning or suspensions. We conduct saware, s-oblivious, and spin-based analysis of pi-blocking under the RNLP.

Unlike group locks, the RNLP does not require resources to be statically grouped before execution. All that is required is a partial order on resource acquisitions, which is a common assumption in practice to ensure that deadlock is impossible.

Organization. In Sec. 2, we formally describe our system model and assumptions. In Sec. 3 we describe the basic architecture of the RNLP, which is composed of two components. In Secs. 4 and 5, we describe and analyze each of these components. We then give provide a brief discussion of the benefits of fine-grained locking in Sec. 6 and conclude in Sec. 7.

\section{Background and Definitions}

We consider a system of $n$ sporadic tasks $\tau=\left\{T_{1}, \ldots, T_{n}\right\}$ that execute on $m$ processors. Each task $T_{i}$ is composed of a sequence of jobs; we let $J_{i, j}$ denote the $j^{\text {th }}$ job of the $i^{t h}$ task. We omit the job index $j$ if it is insignificant. Each task is

\footnotetext{
${ }^{1} \mathrm{~S}$-aware optimality in clustered systems is still an open issue, as discussed in Sec. 5 .

${ }^{2} k$-exclusion generalizes mutual exclusion by allowing up to $k$ simultaneous lock holders.
}

characterized by a worst-case execution time $e_{i}$, a minimum job separation $p_{i}$, and a relative deadline $d_{i}$. For simplicity, we assume for each $T_{i}$ that $p_{i}=d_{i}$ and that each job of $T_{i}$ must complete before its deadline (no tardiness); these assumptions have no bearing on any optimality claims made in this paper. A job is said to be released, when it is made available for execution, and it is pending until it finishes its execution.

Resources. We assume a similar resource model to that presented by Brandenburg and Anderson [4]. The system contains $q$ shared resources $\mathcal{L}=\left\{\ell_{1}, \ldots, \ell_{q}\right\}$ such as shared data objects or I/O devices. In this paper, we consider only mutex locks (though we employ a $k$-exclusion lock to realize the locks that we construct), and as such, at most one job can hold each resource $\ell_{a}$ at any time. Access to shared resources is controlled by a locking protocol. When a job $J_{i}$ requires a resource $\ell_{a}$, it makes a request for $\ell_{a}$ to the locking protocol. $J_{i}$ 's request is said to be satisfied when $J_{i}$ acquires $\ell_{a}$, and completes when $J_{i}$ releases $\ell_{a}$. A job that has issued a resource request that has not yet been satisfied is said to have an outstanding resource request. A job that has issued a resource request that is not complete is said to have an incomplete resource request. We let wait $\left(J_{i}, t\right)$ denote the resource for which $J_{i}$ is waiting at time $t$ if any. The segment of a job between one of its requests being satisfied and completed is called a critical section. A job can either spin (i.e., busy-wait) or suspend while waiting for one of its requests to be satisfied. A ready job is one that can be scheduled; thus, a job that is suspended and waiting for a shared resource is not ready.

If $J_{i}$ holds no resources when it makes a request, then the request is said to be an outermost request. We denote $J_{i}$ 's $k^{t h}$ outermost request as $\mathcal{R}_{i, k}$ and the corresponding resource $\mathcal{F}_{i, k}$. Once $J_{i}$ acquires a resource, it may make a nested request for another resource. If $J_{i}$ acquires a resource at time $t$ via an outermost request, and $t^{\prime}$ is the earliest subsequent time when $J_{i}$ holds no resources, then $\left(t, t^{\prime}\right]$ is called an outermost critical section. Note that resource requests do not have to be properly nested, as seen in Fig. 1 (here, $\ell_{a}$ is acquired first, but $\ell_{b}$ is released last). The maximum number of outermost requests $J_{i}$ makes is given by $N_{i}$. The maximum duration of $J_{i}$ 's $k^{t h}$ outermost critical section is $L_{i, k}$. We say that $J_{i}$ 


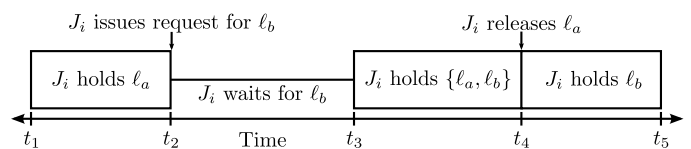

Figure 1: Illustration of a job $J_{i}$ 's outermost critical section. At time $t_{1}, J_{i}$ acquires resource $\ell_{a}$. At time $t_{2}, J_{i}$ issues a nested resource request for $\ell_{b}$, and is blocked during the interval $\left[t_{2}, t_{3}\right)$. At time $t_{4}, J_{i}$ releases $\ell_{a} . J_{i}$ 's outermost critical section spans from $t_{1}$ to $t_{5}$ when $J_{i}$ no longer holds any shared resources.

makes progress if a job that holds a resource for which $J_{i}$ is waiting is scheduled and executing its critical section.

The RNLP requires a strict (irreflexive) partial order, $\prec$, on the set of resources such that a job holding resource $\ell_{b}$ cannot make a resource request for $\ell_{a}$ if $\ell_{a} \prec \ell_{b}$. In addition to preventing deadlock, this ordering is used by the RNLP to improve pi-blocking bounds. There can be a few exceptions to this requirement ${ }^{3}$ that allow for increased concurrency, however none of these exceptions are required to derive worst-case results.

Scheduling. We consider partitioned, clustered, and globally scheduled systems. Under clustered scheduling, the $m$ processors are partitioned into $\frac{m}{c}$ non-overlapping sets of $c$ processors. ${ }^{4}$ Each task is statically assigned to a cluster, and may migrate freely among the processors in the cluster. Partitioned and global scheduling are special cases of clustered scheduling with $c=1$ and $c=m$, respectively.

Within each cluster, jobs are scheduled from a single ready queue using a JLSP scheduling algorithm. In such an algorithm, each job has a base priority. The locking protocols we develop allow a job's base priority to be elevated such that it has a higher effective priority. We denote a job $J_{i}$ 's effective priority at time $t$ as $p\left(J_{i}, t\right)$. Within each cluster, the (at most) $c$ jobs with the highest effective priority are scheduled at any point in time. Three techniques can be used (separately) to elevate a job's effective priority: priority boosting, priority inheritance, and priority donation. A job that is priority boosted has its priority unconditionally raised to ensure it is scheduled. Under priority inheritance, a job executes with an effective priority equal to that of a suspended job. Under priority donation, a higher-priority job $J_{i}$ that, upon release, should preempt some lower-priority job $J_{k}$ with an incomplete resource request instead suspends and donates its priority to $J_{k}$ to ensure that $J_{k}$ makes progress [5]. Upon the completion of $J_{k}$ 's outermost critical section, $J_{i}$ ceases to be a priority donor, and will never be a priority donor again.

Blocking. We adopt the following definition of s-oblivious and s-aware pi-blocking and spin-blocking defined by Brandenburg and Anderson [4, 5, 2]; the first two definitions only apply to suspension-based locks while the third only applies to spin-based locks.

Definition 1. Under s-aware schedulability analysis, a job $J_{i}$ incurs s-aware pi-blocking at time $t$ if $J_{i}$ is pending but

\footnotetext{
${ }^{3}$ Described in more detail in an online appendix available at http: //www. cs. unc. edu/ anderson/papers. html.

${ }^{4}$ Non-uniform cluster sizes could be integrated into our analysis at the expense of more verbose notation.
}
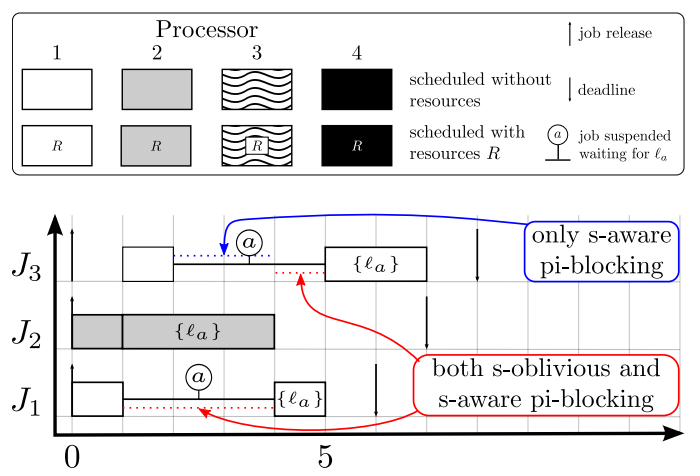

Figure 2: Illustration adapted from [4] of the difference between s-oblivious and s-aware analysis. In this example, three EDFscheduled jobs share a single resource $\ell_{a}$ on two processors. During $[2,4), J_{3}$ is blocked, but there are $m$ jobs with higher priority, thus $J_{3}$ is not s-oblivious pi-blocked. However, because $J_{1}$ is also suspended, $J_{3}$ is s-aware pi-blocked. Intuitively, under s-oblivious analysis, the suspension time of higher-priority jobs is modeled as computation, but under s-aware analysis, it is not. Note the legend applies to all subsequent figures.

not scheduled and fewer than $c$ higher-priority jobs are ready in $T_{i}$ 's cluster.

Definition 2. Under s-oblivious schedulability analysis, a job $J_{i}$ incurs $s$-oblivious pi-blocking at time $t$ if $J_{i}$ is pending but not scheduled and fewer than $c$ higher-priority jobs are pending in $T_{i}$ 's cluster.

Definition 3. A job $J_{i}$ incurs spin-based blocking at time $t$ if $J_{i}$ is spinning (and thus scheduled) waiting for a resource.

The difference between s-oblivious and s-aware piblocking is demonstrated in Fig. 2.

Note that if a job spins non-preemptively then it may cause pi-blocking for jobs that otherwise would have been scheduled. The duration of pi-blocking caused by non-preemptivity must be analyzed in this case as well. Similarly, priority donation and priority boosting may cause jobs that are not currently utilizing a locking protocol to be pi-blocked, and this blocking must be analyzed as well (as we do later).

Similar to $[4,5]$, we measure the blocking behavior of the RNLP using the maximum duration of pi-blocking. However, when supporting nested resource requests, a job can be pi-blocked while holding a resource, as seen in Fig. 1. This "inner" pi-blocking must be included in the analysis of the total duration of pi-blocking. Furthermore, existing analysis of locking protocols is conducted in terms of the maximum critical section length. In our analysis, we instead consider the maximum execution time of a critical section, since the maximum critical section length can depend on the duration of "inner" pi-blocking caused by the locking protocol. The maximum critical section length and the maximum execution time are the same when the RNLP is compared with singleresource locking protocols.

Assumptions. Tight blocking bounds are a function of a number of variables such as the frequency of resource requests, duration of each critical section, and the number of nested requests made. In our asymptotic analysis, we con- 


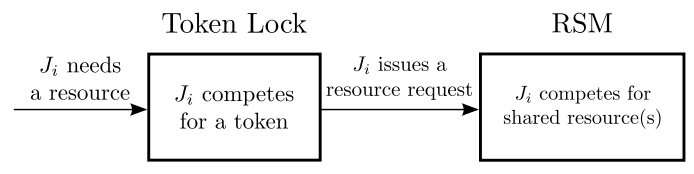

Figure 3: Components of the RNLP.

sider the number of outermost requests per job $N_{i}$, as well as the maximum critical section length $L^{\max }=\max L_{i, k}$ to be constant. We consider both $n$ and $m$ to be variables, and assume $n \geq m$. All other parameters are considered to be constant. Note that we do not impose any restrictions on the number of tasks sharing each resource, the ratio of largest to smallest task period or relative deadline, or the maximum depth of nested requests.

\section{The RNLP}

The RNLP is composed of two components, a $k$-exclusion token lock, and a request satisfaction mechanism (RSM). The token lock restricts the number of jobs that can have an incomplete resource request while the RSM determines when requests are satisfied. In order for a job to issue a resource request, it must first acquire a token through the token lock. The $k$ token-holding jobs can then compete for shared resources according to the rules of the RSM. Depending upon the system (partitioned, clustered, or global), how waiting is realized (suspension or spinning), and the type of analysis being conducted (s-oblivious, s-aware, or spin-based), different token locks, number of tokens (most often $k$ is $n$ or $m$ ), and RSMs can be paired to yield an asymptotically optimal locking protocol supporting nested requests. This architecture is shown in Fig. 3.

We specify the RSM via a set of rules. Without loss of generality, these rules are presented assuming a uniform cluster size of $c$. We assume a few basic properties of the token lock defined as follows (specific token locks are considered in Sec. 5).

T1 There are at most $k$ token-holding jobs at any time, of which there are no more than $c$ from each cluster.

T2 If a job is pi-blocked waiting for a token, then it makes progress. This can be accomplished by elevating the priority of a token-holding job through priority boosting, inheritance, or donation.

Once a job acquires a token, it is allowed to compete for a shared resource under the rules of the RSM. There are several rules and key ideas common to all RSMs. For each shared resource $\ell_{a}$, there is a resource queue $\mathrm{RQ}_{a}$ of length at most $k$. The timestamp of token acquisition is stored for each job $J_{i}$, and denoted $t s\left(J_{i}\right){ }^{5}$ Each resource queue is priority ordered by increasing timestamp. In the absence of any nested resource requests, this ordering is the same as FIFO ordering. This queue ordering allows a job performing a nested resource request to effectively "cut in line" to where it would have been had it requested the nested resource at the time of

\footnotetext{
${ }^{5} \operatorname{ts}\left(J_{i}\right)$ is really a function of time because it is updated for every outermost critical section. However, because we analyze the RNLP on a perrequest basis, we omit the time parameter for notional simplicity.
}

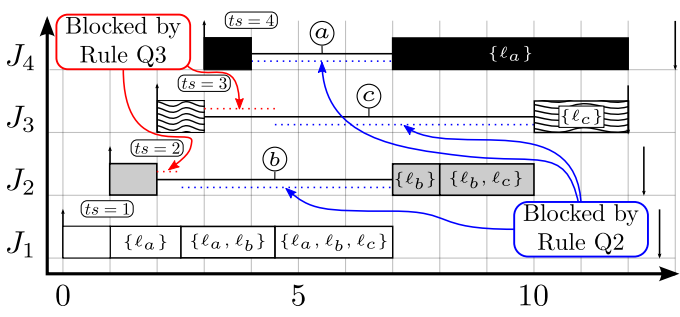

Figure 4: Illustration of Example 1 where $m=4$ and $q=3$.

its outermost resource request. We denote the job at the head of $\mathrm{RQ}_{a}$ as $h d(a)$. The rules below (illustrated below in Example 1) are common to all RSMs.

Q1 When $J_{i}$ acquires a token at time $t$, its timestamp is recorded: $\operatorname{ts}\left(J_{i}\right):=t$. We assume a total order on such timestamps.

Q2 All jobs in $\mathrm{RQ}_{a}$ are waiting with the possible exception of $h d(a)$.

Q3 A job $J_{i}$ acquires resource $\ell_{b}$ when it is the head of the $\mathrm{RQ}_{b}$, i.e., $J_{i}=h d(b)$, and there is no resource $\ell_{a}$ such that $\ell_{a} \prec \ell_{b}$ and $t s(h d(a))<t s\left(J_{i}\right){ }^{6}$

Q4 When a job $J_{i}$ issues a request for resource $\ell_{a}$ it is enqueued in $\mathrm{RQ}_{a}$ in increasing timestamp order. ${ }^{7}$

Q5 When a job releases resource $\ell_{a}$ it is dequeued from $\mathrm{RQ}_{a}$ and the new head of $\mathrm{RQ}_{a}$ can gain access to $\ell_{a}$, subject to Rule Q3.

Q6 When $J_{i}$ completes its outermost critical section, it releases its token.

These rules do not specify how waiting is realized. A specific RSM may employ either spinning or suspending.

Example 1. To illustrate these rules, we present an example, which is depicted in Fig. 4. Consider a global-EDF (G-EDF) scheduled system with three shared resources, $\ell_{a}, \ell_{b}$, and $\ell_{c}$ (so $q=3$ ), and $m=4$, and a total order on resources by index (i.e., $\ell_{a} \prec \ell_{b} \prec \ell_{c}$ ). As shown in Fig. 4, each job $J_{i}$ acquires a token at time $t=i$, and thus by Rule Q1, $\operatorname{ts}\left(J_{i}\right)=i$. Furthermore, $N_{i}=1$, and $\mathcal{F}_{1,1}=\ell_{a}, \mathcal{F}_{2,1}=\ell_{b}, \mathcal{F}_{3,1}=\ell_{c}$, and $\mathcal{F}_{4,1}=\ell_{a}$. At times $t=2.5$ and $t=4.5, J_{1}$ issues nested requests for $\ell_{b}$ and $\ell_{c}$, respectively. These requests are satisfied immediately, because $J_{1}$ has an earlier timestamp than any job in either $\mathrm{RQ}_{b}$ and $\mathrm{RQ}_{c}$. Note that at time $t=3, J_{3}$ has the earliest timestamp of the jobs in $\mathrm{RQ}_{c}$. However, by Rule Q3, $J_{3}$ must wait until $J_{1}$ completes its outermost critical section before it can acquire $\ell_{c}$. Thus, when $J_{1}$ requests $\ell_{c}$ at time $t=4.5, J_{3}$ has not acquired $\ell_{c}$ and hence $J_{1}$ 's request is satisfied immediately. At time $t=7, J_{1}$ finishes its outermost critical section, and $J_{4}$ acquires $\ell_{a}$. Because $J_{2}$ has an earlier timestamp than $J_{4}, J_{2}$ can also acquire $\ell_{b}$ at time $t=7$. However, $J_{3}$ must wait until $t=10$ for $J_{2}$ to finish its outermost critical section before its request for $\ell_{c}$ is satisfied. Note that during the interval $[7,10)$, both $J_{2}$ and

\footnotetext{
${ }^{6}$ As shown in the online appendix, if more information is known about the task set, this rule can be relaxed to allow for more concurrency.

${ }^{7}$ We assume the acquisition of a token and subsequent enqueueing into some $\mathrm{RQ}_{a}$ occur atomically.
} 


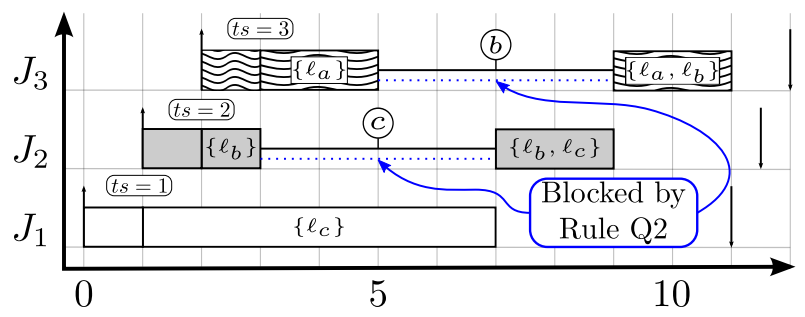

Figure 5: Illustration of Example 2 where $m=4$ and $q=3$.

$J_{4}$ hold shared resources to which a group lock would have required serial access.

To analyze the behavior of an RSM, we first develop terminology and notation to describe when and how jobs can be blocked. Job $J_{i}$ in some resource queue $\mathrm{RQ}_{a}$ is said to be $d i$ rectly blocked by every job before it in $\mathrm{RQ}_{a}$. In our previous example, at time $t=4, J_{4}$ is directly blocked by $J_{1}$ while $J_{1}$ holds resource $\ell_{a}$. The set of jobs that $J_{i}$ is directly blocked by is denoted

$$
D B\left(J_{i}, t\right)=\left\{J_{k} \in \mathrm{RQ}_{\text {wait }\left(J_{i}, t\right)} \mid t s\left(J_{k}\right)<t s\left(J_{i}\right)\right\} .
$$

Note that $J_{i}$ can be directly blocked by at most one resourceholding job $J_{h}$. This is because only one job can hold $\operatorname{wait}\left(J_{i}, t\right)$ at time $t$. It is possible that $J_{h}$ itself is directly blocked by another resource holding job. In this case, all jobs that are blocking $J_{h}$ also block $J_{i}$. We call this transitive blocking. Transitive blocking is the transitive closure of direct blocking. The set of jobs that transitively block $J_{i}$ at time $t$ is given by

$$
T B\left(J_{i}, t\right)=\bigcup_{J_{k} \in D B\left(J_{i}, t\right)} D B\left(J_{k}, t\right) .
$$

Note that $D B\left(J_{i}, t\right) \subseteq T B\left(J_{i}, t\right)$.

Example 2. To illustrate transitive blocking we consider the schedule shown in Fig. 5, which pertains to the same task system as in Example 1. At time $t=1$, job $J_{1}$ acquires $\ell_{c}$, at time $t=2, J_{2}$ acquires $\ell_{b}$, and at time $t=3, J_{3}$ acquires $\ell_{a}$. Also, at time $t=3, J_{2}$ issues a nested resource request for $\ell_{c}$, and at time $t=5, J_{3}$ issues a nested request for $\ell_{b}$. At time $t=5, J_{3}$ is directly blocked by $J_{2}$, and $J_{2}$ is directly blocked by $J_{1}$. Thus, $J_{3}$ is transitively blocked by both $J_{1}$ and $J_{2}$.

Reconsidering Example 1, at time $t \in[3,4.5)$ in Fig. $4, J_{3}$ waits by Rule Q3 even though it is at the head of its resource queue. This gives rise to a different form of blocking that we must also quantify in our analysis. We say that a job that is blocked by a job with an earlier timestamp in another queue is indirectly blocked. The set of jobs that $J_{i}$ is indirectly blocked by at time $t$ is given by

$$
\begin{array}{r}
I B\left(J_{i}, t\right)=\left\{J_{k} \in \mathrm{RQ}_{a} \mid\right. \\
\ell_{a} \prec \operatorname{wait}\left(J_{i}, t\right) \wedge \\
\left.t s\left(J_{k}\right)<t s\left(J_{i}\right)\right\} .
\end{array}
$$

We use the general term blocked to refer to either transitive or indirect blocking. We denote the set of jobs that block $J_{i}$ as

$$
B\left(J_{i}, t\right)=T B\left(J_{i}, t\right) \cup I B\left(J_{i}, t\right) .
$$

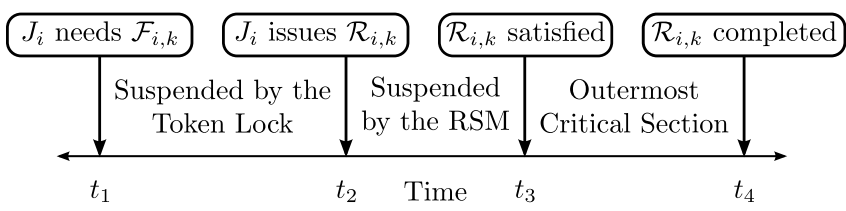

Figure 6: Phases of a resource request in the RNLP.

From the definition of $B\left(J_{i}, t\right)$, we have the following.

Lemma 1. For any job $J_{i}$ and any time $t, \forall J_{k} \in$ $B\left(J_{i}, t\right), \operatorname{ts}\left(J_{k}\right)<t s\left(J_{i}\right)$.

To ensure a bounded duration of pi-blocking, every RSM must satisfy the following property.

P1 If $J_{i}$ is pi-blocked (s-oblivious, s-aware, or spin-based) by the RSM, then $J_{i}$ makes progress.

Properties P1 and T2 combine to ensure that a job that is piblocked waiting for a token makes progress towards acquiring the shared resource it needs. Property P1 will be proved in Sec. 4 for a number of individual RSMs.

Analysis. We now prove a bound on the maximum duration of pi-blocking experienced by a token-holding job $J_{i}$. In the following analysis, let $t_{1}$ denote the time that $J_{i}$ makes a request for a token and $t_{2}$ be the time that $J_{i}$ receives a token. Also, let $t_{3}$ be the time that $J_{i}$ 's outermost request is satisfied and $t_{4}$ be the time that its outermost critical section completes. These times are depicted in Fig. 6.

A job's worst-case duration of pi-blocking is equal to the sum of the maximum duration of pi-blocking caused by the token lock during $\left[t_{1}, t_{2}\right)$ and by the RSM during $\left[t_{2}, t_{3}\right)$ before the $J_{i}$ 's outermost request is satisfied, as well as during $\left[t_{3}, t_{4}\right)$ if $J_{i}$ issues a nested request. We now consider the piblocking caused by the RSM. Later, in Sec. 5 we consider worst-case pi-blocking under various token locks.

Theorem 1. The maximum duration of pi-blocking (regardless of whether waiting is realized by spinning or suspending, or in the latter case if analysis is s-oblivious or $s$-aware), during $\left[t_{2}, t_{4}\right)$ for any $R S M$ is $(k-1) L^{\max }$.

Proof. Property $\mathrm{P} 1$ ensures that if a job is pi-blocked, it makes progress. By Lemma 1, a job can never be pi-blocked by a job with a later timestamp. By Property T1, there are at most $k-1$ jobs with earlier timestamps. Thus, a job can be pi-blocked in any RSM for at most $k-1$ outermost critical sections, each of length at most $L^{\max }$.

\section{Specific Request Satisfaction Mechanisms}

In this section, we describe four request satisfaction mechanisms, the spin RSM (S-RSM), boost RSM (B-RSM), inheritance RSM (I-RSM), and donation RSM (D-RSM).

\subsection{S-RSM}

The S-RSM is the RSM used when waiting is realized by spinning instead of suspending. Spinning is advantageous when critical section lengths are short in comparison to the overhead of a context switch [1,2]. To construct an RSM in which waiting is realized by spinning, we add an additional rule to those common to all RSMs. 
S1 All token-holding jobs execute non-preemptively. A job that is waiting in a resource queue spins.

This rule can be used on partitioned, clustered, or globally scheduled systems. However, there can be no more than $c$ spinning jobs per cluster, and thus there can be at most $k=m$ tokens, $c$ from each cluster. Additionally, non-preemptivity can cause jobs that are not currently utilizing the locking protocol to be pi-blocked. Thus, the execution time of every job must be inflated to account for this possibility.

Lemma 2. The S-RSM for partitioned, clustered, and globally scheduled systems in which waiting is realized by spinning ensures Property $P 1$.

Proof. By Rule S1, every token-holding job is scheduled (and is spinning if it is waiting). Thus, every resource-holding job is scheduled, which ensures that progress is made for all token-holding jobs.

\subsection{B-RSM}

The B-RSM can be applied in partitioned, clustered, or globally scheduled systems in which waiting is realized by suspending instead of spinning. Under the B-RSM, progress is ensured by boosting the priority of a resource-holding job, similar to the $\mathrm{FMLP}^{+}[2]$. Priority boosting, like nonpreemptive spinning, can cause jobs that are not utilizing the locking protocol to be pi-blocked. The following rule defines the B-RSM.

B1 The (at most) $m$ jobs with the earliest timestamps among the resource-holding jobs without outstanding resource requests (i.e., that are not waiting) are boosted above the priority of all non-resource-requesting jobs.

This rule allows for any value of $k$, as it ensures that no more than $m$ jobs can be priority boosted concurrently. The following lemma follows immediately.

Lemma 3. The B-RSM for partitioned, clustered, or globally scheduled systems in which waiting is realized by suspending ensures Property $P 1$.

Note that the B-RSM ensures progress under any JLSP scheduler, but does not always lead to an asymptotically optimal locking protocol due to the pi-blocking boosting can cause on jobs that are not currently utilizing the locking protocol, as described by Brandenburg [2].

\subsection{I-RSM}

The I-RSM is only applicable on globally scheduled systems because it requires that the priorities of all resourcerequesting jobs can be compared. It also requires waiting to be realized by suspending instead of spinning. The I-RSM uses priority inheritance instead of priority boosting as a progress mechanism, which is advantageous because it does not induce pi-blocking on non-resource-requesting jobs.

To motivate the design of the I-RSM, consider again Example 1. Suppose at time $t=6$ there exist $m$ jobs (not shown) that do not utilize the locking protocol that have deadlines just after $t=12$ but before $J_{1}$ 's deadline. Then $J_{3}$ is the only token-holding job that has a sufficient priority to be scheduled. However, $J_{3}$ is blocked by $J_{1}$, which holds $\ell_{c}$, and
$J_{1}$ does not have sufficient priority to be scheduled, and thus is also suspended. $J_{3}$ therefore does not make progress and it can thus have an unbounded duration of pi-blocking. Priority inheritance can be applied to limit such pi-blocking.

We call the job with the earliest timestamp that blocks $J_{i}$ the inheritance candidate of $J_{i}$. The inheritance candidate is thus given by

$$
i c\left(J_{i}, t\right)=\underset{J_{k} \in B\left(J_{i}, t\right)}{\arg \min } t s\left(J_{k}\right) .
$$

A job $J_{c}$ may be the inheritance candidate of several jobs. We define the inheritance candidate set $(I C S)$ of $J_{c}$ to be the set of jobs for which $J_{c}$ is the inheritance candidate.

$$
I C S\left(J_{c}, t\right)=\left\{J_{i} \mid \exists T_{i} \in \tau, i c\left(J_{i}, t\right)=J_{c}\right\} .
$$

In Example 1, at time $t=6, J_{2}, J_{3}$, and $J_{4}$ are all blocked by $J_{1} . J_{1}$ is therefore the inheritance candidate of $J_{2}, J_{3}$, and $J_{4} . J_{1}$ is also the earliest job by timestamp that blocks each of $J_{2}, J_{3}$ and $J_{4}$. Thus, $i c\left(J_{2}, 6\right)=J_{1}$ and $\operatorname{ICS}\left(J_{1}, 6\right)=$ $\left\{J_{2}, J_{3}, J_{4}\right\}$.

The I-RSM builds upon these ideas. If a job is pi-blocked, then the resource-holding job that blocks it, its inheritance candidate, should be scheduled.

I1 A ready job $J_{i}$ holding resource $\ell_{k}$ inherits the highest priority of the jobs for which it is an inheritance candidate:

$$
p\left(J_{i}, t\right)=\max _{J_{k} \in\left\{J_{i}\right\} \cup I C S\left(J_{i}, t\right)} p\left(J_{k}, t\right) .
$$

In Example 1, at time $t=6, \operatorname{ICS}\left(J_{1}, 6\right)=\left\{J_{2}, J_{3}, J_{4}\right\}$. Of these jobs $J_{3}$ has the highest priority, and thus $J_{1}$ inherits the priority of $J_{3}$.

Lemma 4. A job $J_{i}$ 's priority can be inherited by at most one job at a time.

Proof. By construction, $J_{i}$ has at most one inheritance candidate at any time $t$, and thus there is only one job $J_{c}$ for which $J_{i} \in I C S\left(J_{c}, t\right)$. Thus, $J_{i}$ 's priority will be inherited by $J_{c}$ or by no job at all.

Lemma 4 ensures that there are no two jobs executing with the same identity, which is effectively equivalent to a task having two threads. This would break the sporadic task model, and thus the resulting system would not be analyzable using existing schedulability tests.

Lemma 5. For any job $J_{i}, i c\left(J_{i}, t\right)$ is ready.

Proof. By contradiction. Assume that $J_{c}=i c\left(J_{i}, t\right)$. If $J_{c}$ is not ready, then it is suspended by either Rule Q2 or Q3. In either case, by Lemma $1, J_{c}$ is blocked by a job $J_{b}$ with an earlier timestamp. Thus, $J_{i}$ is also blocked by $J_{b}$. This contradicts the fact that $J_{c}$ is $J_{i}$ 's inheritance candidate.

Lemma 6. The I-RSM for globally scheduled systems in which waiting is realized by suspending ensures Property $P 1$.

Proof. If a job $J_{i}$ is pi-blocked (s-oblivious or s-aware) at time $t$, then $J_{i}$ has sufficient priority to be scheduled under either definition of pi-blocking. By Lemma $5, i c\left(J_{i}, t\right)$ is ready. By 
Rule I1, $i c\left(J_{i}, t\right)$ has priority $p\left(i c\left(J_{i}, t\right), t\right) \geq p\left(J_{i}, t\right)$, and thus $i c\left(J_{i}, t\right)$ is scheduled.

The I-RSM does not place any restrictions on the number of tokens in the system, i.e., the value of $k$. Depending upon the scheduler, analysis type, and token lock, $k$ can be chosen to allow for increased parallelism or decreased worst-case piblocking. This issue is considered in Sec. 5.

\subsection{D-RSM}

The D-RSM is designed for clustered (and hence global and partitioned) systems in which waiting is realized by suspending. In these systems, the I-RSM is not sufficient to ensure progress because priorities cannot be compared across clusters. A job in one cluster therefore cannot inherit the priority of a job in another cluster. In clustered systems, progress can be ensured through priority donation, which prevents problematic preemptions of resource-requesting jobs [5]. For instance, in Example 1, if an additional job $J_{5}$ were released at time $t=7$ with a deadline of $t=11$, then it would donate its priority to $J_{4}$, the lowest priority job, which has an incomplete resource request. There are no new rules for the D-RSM, however the D-RSM does require an additional constraint on the token lock.

C1 A token-holding job has one of the highest $c$ effective priorities in its cluster.

Because there are at most $m$ jobs that have one of the highest $c$ effective priorities in their cluster, there can be at most $m$ token holding jobs and thus $k \leq m$.

Lemma 7. Property C1 implies Properties P1 on partitioned, clustered, and globally scheduled systems in which waiting is realized by suspending.

Proof. Property $\mathrm{C} 1$ ensures that a token holding job has a sufficient effective priority to be scheduled. Thus, a ready resource-holding job (which necessarily holds a token) is scheduled, and progress is ensured.

The D-RSM itself does not cause pi-blocking for nonresource-requesting jobs. However, as we shall see, a token lock that satisfies Property $\mathrm{C} 1$ can cause non-resourcerequesting jobs to be pi-blocked.

\section{Token Locks}

In this section, we describe how existing $k$-exclusion locking protocols can be used as token locks. For each token lock, we describe the best choice of $k$, how to pair the token lock with an RSM, and the analytical worst-case pi-blocking complexity of the complete resulting locking protocol. The results of this section are summarized in Table 2.

\subsection{Spin $k$-exclusion}

When waiting is realized by non-preemptive spinning as in the S-RSM, the best choice of token lock is essentially no token lock at all, because the S-RSM alone upholds the properties of both an RSM as well as a token lock. We call this token lock the trivial token lock (TTL) because a job acquires a token immediately upon request. Because there can be at most $m$ jobs running non-preemptively on $m$ processors, $k=m$ under the TTL. For the remainder of this subsection, we assume $k=m$.

Lemma 8. Properties $T 1$ and $T 2$ are ensured by Rule $S 1$.

Proof. By Rule S1, once a job issues a resource request, it runs non-preemptively until it finishes its outermost critical section. No more than $m$ jobs can therefore have incomplete resource requests at a time. This ensures Property T1. Property T2 is ensured because all jobs with incomplete resource requests are scheduled, and thus make progress.

The non-preemptive nature of spin-locks, just like priority boosting and priority donation, can cause a job to be piblocked even when it has no incomplete resource request.

Theorem 2. Any job in the system can be pi-blocked by a job spinning non-preemptively for a duration of at most $m L^{\max }$.

Proof. In the worst case, there can be $m$ jobs that are not currently utilizing the locking protocol that have sufficient priority to be scheduled but are not, due to $m$ other jobs spinning non-preemptively. All $m$ of the token holding jobs must complete their outermost critical sections before the $m^{\text {th }}$ blocked job can be scheduled.

Theorem 3. The maximum duration of s-blocking perrequest in the $S$-RSM is $(m-1) L^{\max }$.

Proof. Follows from Theorem 1 and $k=m$.

\subsection{CK-OMLP for Clustered Systems}

The most versatile of existing $k$-exclusion locking protocols is the clustered $k$-exclusion OMLP (CK-OMLP) developed by Brandenburg and Anderson [5]. The CK-OMLP can be employed on partitioned, clustered, and globally scheduled systems in which waiting is realized by suspending, and it has asymptotically optimal s-oblivious pi-blocking behavior on all such systems. The CK-OMLP relies upon priority donation to ensure progress, and thus every job with an incomplete resource request has one of the $c$ highest priorities in its cluster. In the remainder of this section, we assume that $k=m$, which ensures that a job is not pi-blocked waiting for a token (while priority donors pi-block, the donation mechanism ensures that the [up to] $m$ token holders have the highest effective priorities in the system).

Lemma 9. The CK-OMLP ensures Properties T1, T2, and $C 1$.

Proof. The CK-OMLP is a $k$-exclusion locking protocol, and thus satisfies Property T1. Lemma 1 of [5] proves that a token-holding job (i.e., a job that is "within its critical section" from the CK-OMLP's perspective) has sufficient priority to be scheduled, which yields Property C1. By Lemma 7, a token holding job makes progress. Thus, a job waiting for a token makes progress, satisfying Property T2.

Under priority donation any job can be forced to donate its priority on job release for a period of time. If a job issues many resource requests, the amortized cost per request is reduced. However, because any job can be pi-blocked while it is a priority donor, every job must inflate its execution cost. Because priorities cannot be compared across clusters, we believe this donation cost for all jobs is fundamental on clus- 


\begin{tabular}{|c|c|c|c|c|c|c|}
\hline Analysis & Scheduler & Token Lock & $k$ & RSM & Every Job Pi-blocking & Per-Request Pi-blocking \\
\hline spin & Any & TTL & $m$ & S-RSM & $m L^{\text {max }}$ & $(m-1) L^{\text {max }}$ \\
\hline \multirow{4}{*}{ s-aware } & Partitioned & TTL & $n$ & B-RSM & $n L^{\text {max }}$ & $(n-1) L^{\text {max }}$ \\
\cline { 2 - 7 } & Clustered & TTL & $n$ & B-RSM & $O(\phi \cdot n)$ & $(n-1) L^{\text {max }}$ \\
\cline { 2 - 7 } & Global $^{\dagger}$ & TTL & $n$ & I-RSM & $O(n)$ & $(n-1) L^{\text {max }}$ \\
\hline \multirow{5}{*}{ s-oblivious } & Partitioned & CK-OMLP & $m$ & D-RSM & $m L^{\text {max }}$ & $(m-1) L^{\text {max }}$ \\
\cline { 2 - 7 } & Clustered & CK-OMLP & $m$ & D-RSM & $m L^{\text {max }}$ & $(m-1) L^{\text {max }}$ \\
\cline { 2 - 7 } & \multirow{3}{*}{ Global } & CK-OMLP & $m$ & D-RSM & $m L^{\text {max }}$ & $(5 m-1) L^{\text {max }}$ \\
\cline { 3 - 7 } & & O-KGLP & $m$ & I-RSM & 0 & $(2 m-1) L^{\text {max }}$ \\
\cline { 2 - 6 } & I-KGLP & $m$ & I-RSM & 0 & \\
\hline
\end{tabular}

${ }^{\dagger}$ Applicable only under certain schedulers as discussed in Sec. 5.4.

Table 2: Configuration and worst-case pi-blocking of the RNLP on various platforms. The columns "Token Lock" and "RSM" describe an instantiation of the RNLP that pairs a token lock with an RSM. The remaining columns show the blocking complexity of the resulting locking protocol under the given assumptions just as in Table 1. All listed protocols are asymptotically optimal except the case of clustered schedulers under s-aware analysis for which no asymptotically optimal locking protocol is known.

tered systems.

Theorem 4. The maximum duration of s-oblivious piblocking per job (regardless of whether the job ever issues a resource request) caused by the donation mechanism of the CK-OMLP is $m L^{\max }$.

Proof. By Lemma 1 of [5], a token-holding job has sufficient priority to be scheduled. By Lemma 2 of [5] and the assumption that $k=m$, the maximum duration of s-oblivious piblocking caused by priority donation is bounded by the maximum amount of time a job (the donee) can hold a token. A job can hold a token for $L^{\max }$ time while it holds shared resource(s), plus $(k-1) L^{\max }$ time by Theorem 1 . Since we assume $k=m$, the maximum duration of pi-blocking caused by priority donation is thus $m L^{\max }$.

Theorem 5. The maximum duration of s-oblivious piblocking per outermost resource request is $(m-1) L^{\max }$ under the D-RSM and CK-OMLP.

Proof. Follows from Theorem 1 and $k=m$.

Theorems 4 and 5 show that the RNLP with the D-RSM and CK-OMLP has the same s-oblivious pi-blocking bound as a mutex lock in the clustered OMLP, as seen in Table 1. However, the RNLP supports nested locking while the OMLP does not. Also note that while the D-RSM and the CK-OMLP produce the same blocking bounds as the TTL and the SRSM, the former produces a suspension-based lock while the latter produces a spin-based lock. A system designer may choose one over the other depending upon critical section lengths and system overheads.

\subsection{O-KGLP for Globally Scheduled Systems}

The CK-OMLP can be used on any system with nonoverlapping clusters. However, any job can be forced to suspend on release to donate its priority by Theorem 4. An application with a mix of non-resource-requesting jobs with small periods and jobs with long periods and long critical sections may therefore be unschedulable as a short-period job may be forced to donate its priority for longer than its period.

Elliott and Anderson developed a $k$-exclusion locking protocol specifically for globally scheduled systems called the
O-KGLP [8]. In the O-KGLP, non-resource-requesting jobs are not affected by the behavior of the locking protocol. The O-KGLP has $O(m / k)$ worst-case s-oblivious pi-blocking, which is asymptotically optimal.

\section{Lemma 10. The $O-K G L P$ ensures Properties $T 1$ and $T 2$.}

Proof. The O-KGLP is a $k$-exclusion lock, and thus satisfies Property T1. By Theorem 1 of [8], the O-KGLP is asymptotically optimal, and progress is ensured because of the bounded duration of pi-block in each component of the O-KGLP by Lemmas 2-5 and 7 of [8]. Thus Property T2 holds under the O-KGLP.

Theorem 6. When the O-KGLP is used in tandem with the I-RSM, the maximum s-oblivious pi-blocking per resource request is given by $(2 m+3 k-1) L^{\max }$.

Proof. From Lemmas 2-5 and 7 of [8], the maximum piblocking per token request is $2 m / k+2$ times the maximum duration of time a job can hold a token (i.e., the "maximum critical section length" from the perspective of the O-KGLP). By Lemma 1 (of this paper), a token-holding job can be blocked by $k-1$ requests, and thus the maximum duration of time a job can hold a token is $k L^{\max }$. Thus, the total time a job can be pi-blocked waiting for a token per request is $(2 m+2 k) L^{\max }$. By Theorem 1 , a job can be pi-blocked by the RSM for $(k-1) L^{\max }$ time per request. Thus, the total pi-blocking per request is $(2 m+3 k-1) L^{\max }$.

The CK-OMLP has a smaller constant factor for the maximum per-request pi-blocking, but the O-KGLP does not require a non-resource-requesting job to inflate its execution time. $^{8}$

\subsection{Trivial Token Lock for S-Aware Analysis}

The token locks discussed above lead to asymptotically optimal implementations under spin-based or s-oblivious analysis. However, these locking protocols do not perform well under s-aware analysis. Under s-aware analysis, it is best to choose $k=n$ to allow for maximal concurrency, thus the

\footnotetext{
${ }^{8}$ In an online appendix, we present a new $k$-exclusion locking protocol (the I-KGLP in Table 2) for globally scheduled systems, that has a better s-oblivious pi-blocking bound under s-oblivious analysis than the O-KGLP.
} 
TTL is used. Note that increased concurrency results in fewer suspensions, which are accounted for under s-aware analysis. From the TTL, we have the following lemma.

\section{Lemma 11. The TTL satisfies Properties $T 1$ and T2.}

Depending upon the scheduler, the TTL can be paired with different RSMs. We pair the TTL with the B-RSM under partitioned scheduling.

Theorem 7. On a partitioned system, the maximum duration of $s$-aware pi-blocking per outermost resource request is $(n-$ 1) $L^{\max }$ under the $B-R S M$ and TTL.

Proof. Follows from Theorem 1 and $k=n$.

Under s-aware analysis we must carefully consider the piblocking boosting itself may cause.

Theorem 8. Let $c=1$ and $n_{c}$ be the number of tasks assigned to $J_{i}$ 's partition. The worst-case s-aware pi-blocking of job $J_{i}$ caused by the boosting of other jobs in the B-RSM (regardless of whether $J_{i}$ ever issues a resource request) is $\left(n_{c}-1\right) L^{\max }$.

Proof. In the worst case, all requests are serialized by the B-RSM, as any concurrency would decrease s-aware piblocking. In this case, the blocking behavior is the same as the $\mathrm{FMLP}^{+}$because the B-RSM employs the same progress mechanism as the $\mathrm{FMLP}^{+}$. Thus, the bound follows directly from the bound for the FMLP ${ }^{+}$in Theorem 6.4 of [2].

Note that the per-job and per-request pi-blocking are both $O(n)$ and thus the pairing of the TTL and the B-RSM is asymptotically optimal for partitioned systems (given the previously established $\Omega(n)$ lower bound [4]).

The B-RSM can be employed in systems in which $c>1$ (including $c=m$ ) and progress is ensured. However, the BRSM can cause a job that is not currently utilizing the locking protocol to be pi-blocked for longer than $O(n)$. Brandenburg showed a lower bound of $\Omega(\phi)$ on s-aware pi-blocking caused by priority boosting where $\phi$ is the ratio of the maximum period to the minimum period in the system [2]. This bound is not proven to be tight. In an online appendix, we establish a coarse upper bound on s-aware pi-blocking under the BRSM where $c>1$ to be $O(\phi \cdot n)$. This bound is based on the number of other jobs that can execute during one job's period.

Priority donation and priority inheritance are the only remaining progress mechanisms to consider. Priority donation is not particularly effective under s-aware analysis [2]. Priority inheritance is only applicable on globally scheduled systems and in general it has the same $\Omega(\phi)$ s-aware pi-blocking bound as priority boosting [2]. However, under rate monotonic (RM) scheduling, as well as constrained, fixed prioritypoint schedulers (e.g., FIFO, and G-EDF with relative deadlines at most periods), a special class of JLSP schedulers in which each task's relative priority point does not exceed its period, priority inheritance yields an asymptotically optimal locking protocol.

Theorem 9. The worst-case s-aware pi-blocking per job (regardless of whether the job ever issues a resource request) caused by the TTL and I-RSM under either RM or any constrained, fixed priority-point global scheduler is $O(n)$.
Proof. In the worst case, all requests are serialized by the I-RSM as any concurrency would decrease s-aware piblocking. In this case, the I-RSM is equivalent to the FMLP. From [2], the maximum s-aware pi-blocking caused by priority inheritance is $O(n)$ for RM and constrained, fixed priority-point global schedulers.

Theorem 10. The maximum duration of s-aware pi-blocking per outermost resource request is $(n-1) L^{\max }$ under the TTL and I-RSM.

Proof. Follows from Theorem 1 and $k=n$.

Note that asymptotically, the RNLP performs no worse than any existing locking protocols under s-aware analysis. However, the increased concurrency afforded by the RSM leads to improved s-aware pi-blocking in practice.

\section{Benefits of Fine-Grained Locking}

The worst-case blocking behavior of the RNLP family of locking protocols is no worse than the FMLP or the OMLP family of locking protocols under the analysis assumptions we have made. However, the RNLP can allow multiple jobs to access resources within a group concurrently. In many applications, nested resource requests, which force resources to be grouped, though possible, are relatively infrequent [3]. In such cases, the RNLP often allows jobs to hold individual resources in a resource group concurrently. This improves average-case pi-blocking. Additionally, if more information is known a priori about a task set, then it may be possible to achieve tighter blocking bounds.

As an example, consider an unmanned aerial vehicle that employs planning algorithms to compute a route through enemy territory to avoid danger while carrying out its mission. Some such algorithms employ imprecise computations [9], in which results improve given more computation time [10]. In such applications, imprecise computation may be used to compute navigation plans for segments of the vehicle's flight path. Later, any spare computational resources can be used to optimize the plans for dangerous segments of the flight path.

To support such an application, we can envision navigation plans being computed by producer task(s), and enqueued into a circular buffer as seen in Fig. 7. When the time comes for a plan to be executed, a consumer task dequeues the plan from the circular buffer. Other tasks can further optimize plans that are already in the circular buffer.

To support this application under group locks, access to any part of the circular buffer would necessarily be arbitrated by a single group lock. Using fine-grained locking with the RNLP, jobs can concurrently modify different parts of the buffer. In this locking scheme, the head and tail pointers are each considered individual shared resources to ensure jobs cannot either produce or consume concurrently. Each element in the buffer is also controlled by a mutex lock to ensure that a plan is not modified concurrently. In this example, to enqueue (dequeue) a plan to (from) the queue, a job must acquire the head (tail) pointer as well as the lock for the element to which the head or tail pointer points.

Under the RNLP, if the buffer is neither empty nor full, then a producer task can never be blocked by a consumer 


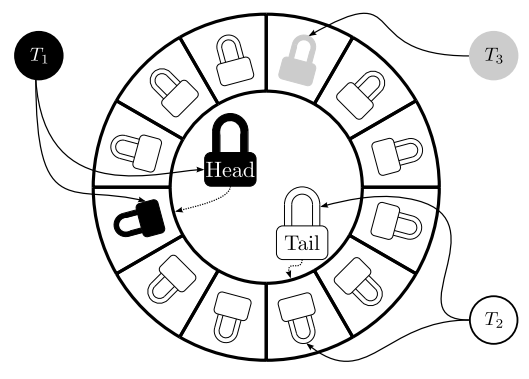

Figure 7: Illustration of a circular buffer for imprecise computation. The head (tail) resource as well as the element at the head (tail) of the queue are locked by one task, while another task has an element in the middle of the queue locked.

task or vice versa. This application-specific knowledge can be incorporated into finer-grained analysis to achieve tighter blocking bounds. In this case, the blocking bound for the RNLP would be less than that of a similar group lock.

Even in applications in which a priori fine-grained locking properties are not known, it can still be advantageous to use the RNLP over a single-resource locking protocol. For example, in a soft real-time system, the increased parallelism afforded by the RNLP may enable more jobs to meet their deadlines. In systems supporting a mix of hard and soft realtime tasks as well as best-effort work, the increased parallelism may allow more best effort work to get done. Even in a hard real-time setting, the RNLP has a benefit; by reducing the duration of pi-blocking, safety margins are made wider to help ensure anomalous behavior does not cause a job to miss its deadline.

\section{Conclusions}

Existing locking protocols for multiprocessor real-time systems only support a single resource. Nested resource requests are therefore only supported through the use of coarsegrained locking techniques such as group locks. In this paper, we have presented the RNLP, a modular locking protocol composed of a $k$-exclusion token lock and an RSM. Token locks and RSMs are paired depending upon the scheduler, type of analysis, and how waiting is realized to achieve asymptotically optimal locking protocols in most cases (and all cases in which asymptotically optimal single-resource locking protocols are known). Furthermore, the modular nature of the RNLP allows for future progress mechanisms or $k$-exclusion locks to be incorporated into the RNLP to improve performance in particular cases.

The variants of the RNLP we have presented have soblivious, s-aware, and spin-based blocking behavior no worse than existing multiprocessor locking protocols under the analysis assumptions we have employed. The RNLP, however, supports nested resource requests, and it is possible for multiple jobs to concurrently hold separate resources in a resource group. This increased parallelism can be advantageous in many settings. The RNLP is also advantageous in that groups do not have to be statically assigned before execution. Resources can be dynamically added or removed so long as the relative order of all other resources is not modi- fied. This property adds flexibility to the RNLP.

This work forms a strong platform on which we intend to continue to build. For example, while this paper only addresses mutex locks, we would like to extend the RNLP to support $k$-exclusion and reader-writer resources, and allow nested requests between all of these types of resources. Additionally, we intend to explore the possibility of dynamic group locks, in which a job can request an arbitrary set of shared resources at once (under the RNLP, a job would have to request each resource in the set individually).

Under the RNLP, all resources are controlled by a single token lock. However, this can cause a job to be blocked waiting for a token held by a job waiting for another resource, possibly one in which critical section lengths are longer. We intend to investigate the possibility of allowing multiple instances of the RNLP to be instantiated to control fine-grained nested requests within individual resource groups, to eliminate the possibility of such an occurrence. Such a scheme would be a hybrid of coarse-grained group locking and finegrained locking.

Also, we want to conduct a more rigorous analysis of the blocking behavior of the RNLP using additional information about a system, such as the semantics of the operations on shared data structures, maximum depth of nested resource requests, the length of each task's critical sections, and the request order. Finally, we plan to implement the RNLP in LITMUS $^{\mathrm{RT}}$ and conduct an empirical evaluation.

Acknowledgements. We thank Glenn Elliott for his helpful discussions pertaining to $k$-exclusion locking protocols.

\section{References}

[1] A. Block, H. Leontyev, B.B. Brandenburg, and J.H. Anderson. A flexible real-time locking protocol for multiprocessors. In RTCSA '07, pages 47-56, Aug. 2007.

[2] B.B. Brandenburg. Scheduling and Locking in Multiprocessor RealTime Operating Systems. $\mathrm{PhD}$ thesis, The University of North Carolina at Chapel Hill, 2011.

[3] B.B. Brandenburg and J.H. Anderson. Feather-trace: A light-weight event tracing toolkit. In OSPERT'07, pages 61-70, 2007.

[4] B.B. Brandenburg and J.H. Anderson. Optimality results for multiprocessor real-time locking. In RTSS '10, pages 49-60, 2010.

[5] B.B. Brandenburg and J.H. Anderson. Real-time resource-sharing under clustered scheduling: Mutex, reader-writer, and k-exclusion locks. In EMSOFT'11, pages 69-78, Sep. 2011.

[6] C.-M Chen and S.K. Tripathi. Multiprocessor priority ceiling based protocols. Technical Report CS-TR-3252, Univ. of Maryland, College Park, 1994.

[7] A Easwaran and B. Andersson. Resource sharing in global fixedpriority preemptive multiprocessor scheduling. In RTSS '09, pages 377-386, 2009.

[8] G.A. Elliott and J.H. Anderson. An optimal $k$-exclusion real-time locking protocol motivated by multi-GPU systems. In RTNS '11, pages 15-24, Sep. 2011

[9] J.W.S. Liu, K.-J. Lin, W.-K. Shih, A.C.-S. Yu, J.-Y. Chung, and W. Zhao. Algorithms for scheduling imprecise computations. Computer, 24(5):58-68, May 1991.

[10] M.S. Mollison, J.P. Erickson, J.H. Anderson, S.K. Baruah, and J.A. Scoredos. Mixed-criticality real-time scheduling for multicore systems. In CIT'10, pages 1864-1871, Jul. 2010.

[11] R. Rajkumar. Synchronization In Real-Time Systems - A Priority Inheritance Approach. Kluwer Academic Publishers, Boston, 1991. 